\title{
Assessment of cough strength in patients with a tracheostomy
}

\author{
Stephen Kowalski, MD, FRCPC (D) - Kandy Macaulay, RRT • \\ Rhonda Thorkelsson, RN, CNCC $\cdot$ Linda Girling, BSc Hons $\cdot$ Zoheir Bshouty, MD, FRCPC
}

Received: 1 May 2017/Revised: 14 August 2017/Accepted: 24 August 2017 /Published online: 5 September 2017

(C) Canadian Anesthesiologists' Society 2017

\section{To the Editor,}

A patient's weak cough has been identified as a risk factor for re-intubation. ${ }^{1}$ It may also impair weaning in patients with a tracheostomy. ${ }^{2}$ The study described herein assessed whether cough strength is enhanced along with tracheal cuff deflation with a one-way valve. This randomized crossover study of mechanically ventilated, tracheostomized patients in the intermediate intensive care unit was registered with Clinical Trials.gov (NCT02566512).

The University of Manitoba Health Research Board approved the study (May, 2016), and all patients provided informed written consent. The study was conducted between September 2015 and January 2017. A convenience sample of 20 patients was chosen. The peak expiratory flow rate (PEFR) while coughing was measured by a respiratory therapist using an air zone peak expiratory flow meter. With the tracheostomy cuff inflated, the PEFR

Trial registration: www.clinicaltrials.gov, NCT 02566512. Registered June 23, 2015.

S. Kowalski, MD, FRCPC ( $) \cdot$ L. Girling, BSc Hons

Section of Critical Care, Department of Anesthesia and

Perioperative Medicine, University of Manitoba, Winnipeg, MB,

Canada

e-mail: sekowalski@hsc.mb.ca

K. Macaulay, RRT

Department of Respiratory Therapy, Health Sciences Centre,

University of Manitoba, Winnipeg, MB, Canada

R. Thorkelsson, RN, CNCC

Department of Nursing, Health Sciences Centre, University of Manitoba, Winnipeg, MB, Canada

Z. Bshouty, MD, FRCPC

Department of Medicine, Section of Critical Care and

Respirology, University of Manitoba, Winnipeg, MB, Canada meter was attached to the tracheostomy tube. The patient was instructed to take as deep a breath as possible and then cough. With the tracheostomy cuff deflated, a one-way valve (Shiley speaking valve) was attached to the tracheostomy tube. The patient used a mouthpiece that was connected to the PEFR meter. Nose clips were applied. The patient was again instructed to take as deep a breath as possible and then cough. All measurements were performed four times and averaged. Patients were randomized to begin with either the tracheal cuff inflated as the first intervention (group 1) or with the tracheal cuff deflated as the first intervention (group 2).

Data, reported as mean (standard deviation), were compared using a paired Student's $t$ test. A value of $P<0.05$ was considered statistical significant.

Twenty patients were enrolled in the study. Their mean (standard deviation [SD]) age was 64 (13) yr, and they had been on mechanical ventilation for 64 (20) days. Their mean (SD) baseline vital capacity was $1,061(337) \mathrm{mL}$ and maximum inspiratory pressure was $-29.5(9.4) \mathrm{cm} \mathrm{H}_{2} \mathrm{O}$.

Among the 20 patients, 19 had a greater PEFR when coughing with the tracheostomy cuff deflated (Figure). The mean (SD) PEFR with the cuff deflated and a one-way valve was $38 \%$ greater than when coughing through the tracheostomy tube $\left[161\right.$ (58) vs 100 (48) L- $\left.\mathrm{min}^{-1}\right]$. The randomization order had no effect on outcome. The patient whose flow rate was lower with the cuff deflated was subsequently found to have subglottic stenosis.

Among patients who require mechanical ventilation, 5$15 \%$ exhibit a prolonged time for liberation from mechanical ventilation, and most ultimately require tracheostomy. Hernandez et al. showed that patients were liberated more quickly when the tracheal cuff was deflated during spontaneous breathing trials, and they had a lower incidence of pulmonary infections. ${ }^{3}$ 


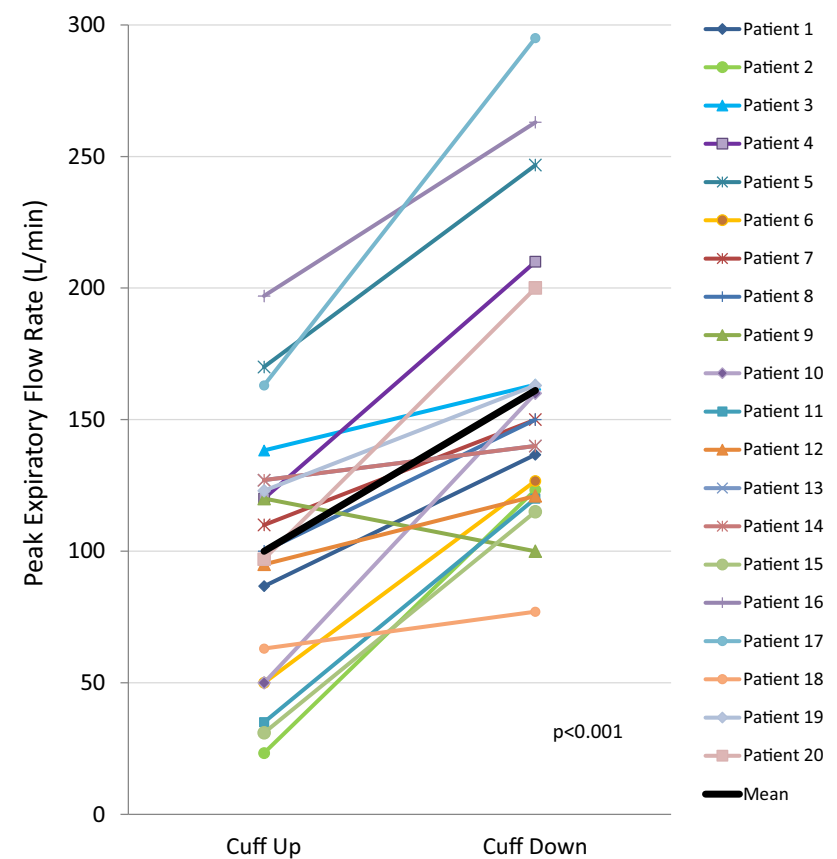

Figure Cough Strength

Our study showed that patients' cough strength increases when they can use their glottis during tracheal cuff deflation in conjunction with a one-way valve. Intubated patients and those breathing via a tracheostomy cannot make use of their glottis and are at a mechanical disadvantage. Hence, they have less effective coughs. Other benefits of cuff deflation include vocalization, facilitation of swallowing and eating, and less need for suctioning because of a stronger cough.

A weak cough was previously identified as a risk factor for re-intubation. Smina et al. showed that intubated patients with a peak expiratory flow rate $<60 \mathrm{~L} \cdot \mathrm{min}^{-1}$ were five times more likely to require re-intubation and 19 times more likely to die during their hospital admission. ${ }^{1}$ Other studies have found that a PEFR of $<60 \mathrm{~L} \cdot \mathrm{min}^{-1}$ is associated with an increased risk of re-intubation., ${ }^{2,4,5}$ Among the 20 patients in the current study, five had a PEFR that was less than the critical value for intubated patients $\left(60 \mathrm{~L} \cdot \mathrm{min}^{-1}\right)$ when the tracheostomy cuff was inflated.

These results suggest there may be an advantage to deflating the tracheostomy tube cuff to facilitate weaning because it allows a stronger cough, better clearance of secretions, and decreased infections. ${ }^{3}$

Conflicts of interest None declared.

Editorial responsibility This submission was handled by Dr. Gregory L. Bryson, Deputy Editor-in-Chief, Canadian Journal of Anesthesia.

Funding This study was supported by a grant from The Academic Oversight Committee of the Department of Anesthesia and Perioperative Medicine, University of Manitoba.

\section{References}

1. Smina M, Salam A, Khamiees M, Gada P, Amoateng-Adjepong $Y$, Manthous $C$. Cough peak flows and extubation outcomes. Chest 2003; 124: 262-8.

2. Perren A, Brochard L. Managing the apparent and hidden difficulties of weaning from mechanical ventilation. Intensive Care Med 2013; 39: 1885-95.

3. Hernandez G, Pedrosa A, Ortiz R, et al. The effects of increasing airway diameter on weaning from mechanical ventilation in tracheostomized patients: a randomized trial. Intensive Care Med 2013; 39: 1063-70.

4. Su WL, Chen $Y H$, Chen $C W$, et al. Involuntary cough strength and extubation outcomes for patients in an ICU. Chest 2010; 137: 77782.

5. Duan J, Liu J, Xiao M, Yang X, Wu J, Zhou L. Voluntary is better than involuntary cough peak flow for predicting re-intubation after scheduled extubation in cooperative subjects. Respir Care 2014; 59: 1643-51. 